\title{
Accurate Prediction of Transimpedances and Equivalent Input Noise Current Densities of Tuned Optical Receiver Front Ends
}

\author{
Liu, Qing Zhong
}

Published in:

Proceedings of the 21st European Microwave Conference

Link to article, DOI:

10.1109/EUMA.1991.336529

Publication date:

1991

Document Version

Publisher's PDF, also known as Version of record

Link back to DTU Orbit

Citation (APA):

Liu, Q. Z. (1991). Accurate Prediction of Transimpedances and Equivalent Input Noise Current Densities of Tuned Optical Receiver Front Ends. In Proceedings of the 21st European Microwave Conference (Vol. Volume 2, pp. 1329-1334). IEEE. https://doi.org/10.1109/EUMA.1991.336529

\section{General rights}

Copyright and moral rights for the publications made accessible in the public portal are retained by the authors and/or other copyright owners and it is a condition of accessing publications that users recognise and abide by the legal requirements associated with these rights.

- Users may download and print one copy of any publication from the public portal for the purpose of private study or research.

- You may not further distribute the material or use it for any profit-making activity or commercial gain

- You may freely distribute the URL identifying the publication in the public portal 


\title{
Accurate Prediction of Transimpedances and Equivalent Input Noise Current Densities of Tuned Optical Receiver Front Ends
}

\author{
Qing Zhong Liu
}

\begin{abstract}
Novel analytical expressions have been derived for calculating transimpedances and equivalent input noise current densities of five tuned optical receiver front ends based on PIN diode and MESFETs or HEMTs. Miller's capacitance, which has been omitted in previous studies, has been taken into account. The accuracy of the expressions has been verified by using Touchstone simulator. The agreement between the calculated and simulated front end performances is very good.
\end{abstract}

\section{Introduction}

In optical fiber transmission systems, the optical receiver front end plays an important role [1]. The sensitivity of the receiver is limited by different noise factors. Among these factors, the $\mathrm{f}^{2}$ thermal noise term from FETs channel is a dominating one for high frequency and wideband applications. To suppress $\mathrm{f}^{2}$ noise, different tuning networks have been applied between the photo detector and the FETs preamplifier. Consequently, the receiver sensitivity has been improved significantly. Some analysis and design information on the tuned optical receiver front ends have been reported in [2]-[4]. In order to make further complex computer simulations and optimizations of the front ends, it is necessary to be able to provide a good starting point and to avoid the local minima in the optimizations.

In this paper, we present some novel analytical and simple expressions which can be used to calculate the transimpedances and equivalent input noise current densities of the most widely used tuned front ends based pin diode and FETs. The expressions have been verified by using Touchstone. The comparison between the calculated and simulated front end performances is shown.

\section{Derivation of Expressions}

A diagram of a tuned optical receiver front end based on a pin diode and a FET is shown in Fig.1. The diode is modelled as a current source, a junction capacitance, and a series resistor. The FET is represented by an equivalent circuit, which is valid for both MESFETs and HEMTs. The five different tuning networks are shown in Fig.2 (a)-(e), respectively.

To derive the expression for transimpedance of the front end, we calculate the voltage drop $V_{1}$ on $C_{g s}$ as function of current $I_{S}$. The output voltage $V_{0}$ of the front end amplifier is then determined and finally the transimpedance is obtained by dividing $\mathrm{V}_{\mathrm{o}}$ by $\mathrm{I}_{\mathrm{s}}$.

In [5], an analytical expression was given for calculating the equivalent input noise current density of a $\mathrm{T}$ equivalent transformer tuned front end, where the correlation between the gate and drain noises has been taken into account. Based on that work, we derived the expressions for determining the equivalent input noise current densities of all other tuned front ends.

Center for Broadband Telecommunications

Electromagnetics Institute, Technical University of Denmark

Lyngby Dk-2800 Denmark 
In the present work, Miller's capacitance, which has been omitted in previous studies, has been included. In fact, neglecting the Miller's capacitance results in an over-estimation of transimpedance and underestimation of input noise current density of the front ends. The accuracy of the calculation has also been improved by taking into account of resistance $R_{i n}, R_{g}$ and $R_{s}$.

In the following we will only show the expressions for calculating the transimpedance and equivalent input noise current density of a $\pi$ equivalent transformer tuned front end. The expressions can be applied to other tuned front ends by simple modifications.

It can be shown that the transimpedance of the $\pi$ equivalent transformer tuned front end is given by:

$$
Z_{\mathrm{m}}=\frac{-\mathrm{g}_{\mathrm{m}} R_{\mathrm{ds}} R_{\mathrm{L}} \mathrm{Z}_{\mathrm{gs}} Z_{\mathrm{a}} Z_{2} Z_{4}}{\left[Z_{\mathrm{em}}+Z_{\mathrm{gs}}+Z_{\mathrm{in}}+Z_{s s}\left(1+g_{m} Z_{\mathrm{gs}}\right)\right]\left[R_{\mathrm{is}}+R_{\mathrm{L}}+Z_{s s}\right]\left(Z_{3}+Z_{4}\right) Z_{1}}
$$

where

$$
\begin{aligned}
& Z_{g s}=\frac{1}{j \omega C_{g s}} \quad Z_{g d}=\frac{1}{j \omega C_{g d}^{\prime}} \quad Z_{a}=\frac{1}{j \omega C_{j}} \\
& Z_{p 1=j \omega L_{p 1}} Z_{p 2}=j \omega L_{p 2} \quad Z_{s}=j \omega L_{s} \\
& \mathrm{C}_{\mathrm{gd}}^{\prime}=\left[1+\mathrm{g}_{\mathrm{m}}\left(\mathrm{R}_{\mathrm{dg}} \| \mathrm{R}_{\mathrm{L}}\right)\right] \mathrm{C}_{\mathrm{gd}} \quad \mathrm{Z}_{\mathrm{in}}=\mathrm{R}_{\mathrm{g}}+\mathrm{R}_{\mathrm{in}} \\
& Z_{s \mathrm{~s}}=j \omega l_{\mathrm{s}}+R_{\mathrm{s}} \quad Z_{1}=Z_{\mathrm{a}}+R_{\mathrm{j}} \\
& Z_{2}=Z_{1} \| Z_{p 1} \quad Z_{3}=Z_{2}+Z_{s} \\
& \mathrm{Z}_{4}=\mathrm{Z}_{\mathrm{p} 2}\left\|\mathrm{Z}_{\mathrm{gd}}^{\prime} \quad \mathrm{Z}_{\mathrm{em}}=\mathrm{Z}_{3}\right\| \mathrm{Z}_{4}
\end{aligned}
$$

Since the $\mathrm{f}^{2}$ thermal noise term from FETs channel is a dominating factor for high frequency and wideband applications, we will only consider its contribution in the following. It can be shown that the equivalent input noise current density of the $\pi$ equivalent transformer tuned front end is given by:

$$
\frac{\overline{i^{2}{ }^{2}}}{\Delta f}=\frac{4 K T}{g_{m}} \frac{1}{\left|Z_{21} / A\right|^{2}}\left\{P+R\left|Z_{G O}\right|^{2}\left(\omega C_{g s}\right)^{2}-2 C_{o r} \sqrt{P R}\left(\omega C_{g s} \operatorname{Im}\left[Z_{G O}\right]\right)\right\}
$$

where

$$
\begin{aligned}
& Z_{21}=\frac{Z_{a} Z_{2} Z_{g s} Z_{e m}}{Z_{1} Z_{3}\left[\left(1+g_{m} Z_{g s}\right) Z_{s s}+Z_{i n}+Z_{g s}+Z_{e m}\right]} \\
& Z_{G O}=Z_{g s} \|\left(Z_{e m}+Z_{s s}+Z_{i n}\right) \\
& A=\frac{Z_{s s}+Z_{i n}+Z_{g s}+Z_{e m}}{\left(1+g_{m} Z_{g s}\right) Z_{s s}+Z_{i n}+Z_{g s}+Z_{e m}}
\end{aligned}
$$

Eqs.(1) and (2) can be used for other tuned front end configurations. To calculate the transimpedance and input equivalent noise current density of a serially tuned front end, $Z_{p 1}$ is set to be infinite, and $Z_{p 2}$ is replaced by a load resistance $R_{b}$. A noise term $4 \mathrm{KT} / \mathrm{R}_{\mathrm{b}}$ resulting from $\mathrm{R}_{\mathrm{b}}$ has to be added in the calculation of the noise current density. The expressions for a parallel tuned front end are obtained by letting $L_{s}$ be infinitesimal. By using the well known $\pi$ to $T$ network transformation, transimpedance and equivalent input noise current density of the $T$ equivalent transformer tuned front end can also be calculated. The expressions are obtained for 3rd order bandpass tuned front end by replacing the series inductance $Z_{\mathbf{s}}$ with $Z_{s}+Z_{c}$, where $Z_{c}$ is the reactance of series capacitance $C_{c}$ in the tuning network. 


\section{Comparison Between Calculations And Simulations}

To demonstrate the accuracy of the expressions, the transimpedances and equivalent input noise current densities of five different tuned front ends have been calculated by using the expressions and simulated by using Touchstone simulator, respectively. The photo diode used is a pin diode from BT\&D (PDC 4300) with $\mathrm{C}_{\mathrm{j}}$ of $0.12 \mathrm{pf}$ and $\mathrm{R}_{j}$ of $10 \Omega$. The FET is a HEMT from NEC (NE20200).

In Fig.3 (a)-(e), comparisons between the calculated and simulated transimpedances of the front ends are shown. For a serially tuned front end, the maximum deviation between the calculated and simulated results is about $2.5 \mathrm{~dB}$ from $\mathrm{DC}$ to $12 \mathrm{GHz}$. For all the bandpass tuned front ends, the maximum deviation is less $2 \mathrm{~dB}$. It can be seen that the parallel tuned front end is only suitable for narrow band applications. Compared with the $T$ equivalent transformer and $\pi$ equivalent transformer tuned front ends, the 3rd order bandpass tuned end offers wider bandwidth. In Fig.4 (a)-(e), comparisons between the calculated and simulated equivalent input noise current densities of different front ends are shown. The agreement between the calculated and simulated results is good. The maximum deviation between the calculated and simulated results is less than 2 $\mathrm{pA} / \mathrm{JHz}$ for the serial tuned front end. For the bandpass tuned front ends, the maximum deviations are less than $2.5 \mathrm{pA} / \mathrm{VHz}$. The results shown above are not the optimized ones, and further computer simulations and optimizations are needed to obtain better performances of the front ends for specified applications.

\section{Conclusions}

In this paper, we presented some novel and analytical expressions for calculating the transimpedances and equivalent input noise current densities of five tuned optical receiver front ends based on the PIN diode and FETs. The expressions can be used for analysis of front ends built with either MESFETs or HEMTs. The accuracy of the expressions has been verified by using Touchstone simulator. The expressions will find applications in predicting performances and providing a starting point for simulation and optimization of the optical receiver front ends.

\section{Acknowledgements}

The author wishes to thank Prof. Palle Jeppesen and Dr. Per Danielsen for encouragement and valuable discussions during the work. The author would like also to thank Mr. Morten Høgdahl from the Electromagnetics Institute, Technical University of Denmark, for providing the model for calculating the equivalent input noise current density of the front ends in Touchstone, and Mr. K. E. Alameh from the University of Sydney, Australia, for the discussion on the equivalent input noise current density of the front ends. This work was supported by the Danish Technical Research Council through the Center for Broadband Telecommunications.

\section{References}

[1] S.D.Personick, The Bell System Technical Journal. Vol.52, No.6, 1974, pp.843886.

[2] T E. Darcie et al, J. of Lightwave Technology. Vol.6. No.4. 1988. pp.582-589.

[3] N.Ohkawa, J. of Lightwave Technology. Vol.6. No.11. 1988. pp.1665-1671.

[4] G.Jacobsen et al, J.of Lightwave Technology. Vol.7. No.1. 1989. pp.105-114.

[5] K E. Alameh et al, IEEE Trans. MTT., Vol.38, No. 5, 1990, pp.546-551. 


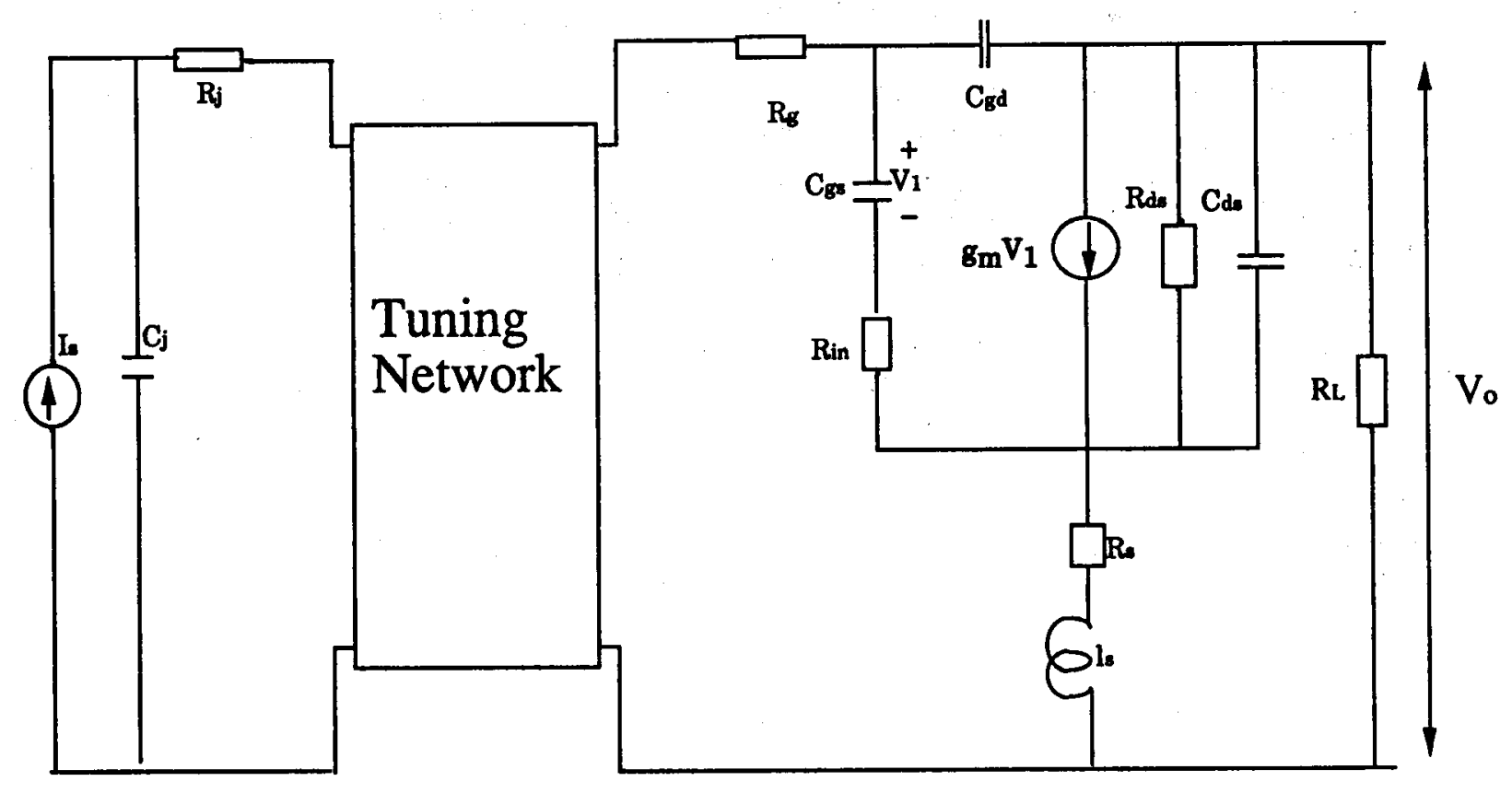

Fig.1 Diagram of a general tumed optical receiver front end based on PIN diode and FET
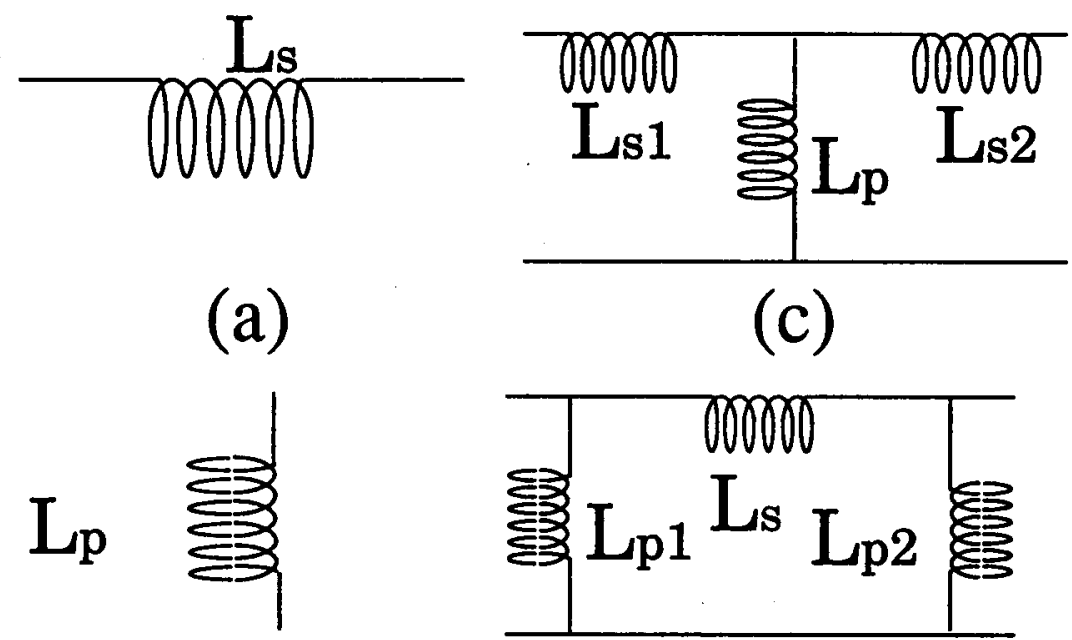

(b)
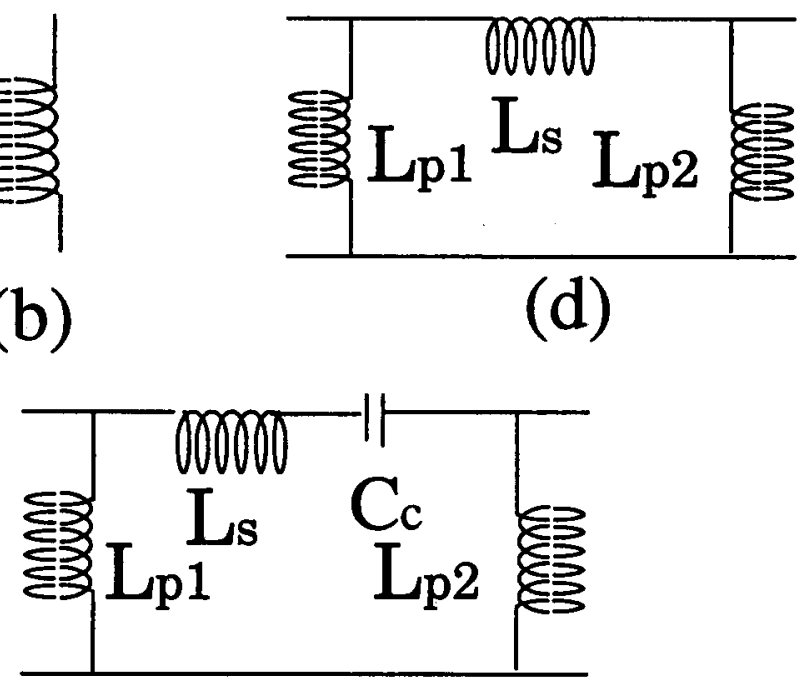

(e)

Fig.2 Tuning networks of (a) serially (b) parallel (c) T-equivalent transformer (d) $\pi$ equivalent transformer (e) 3rd order bandpass 


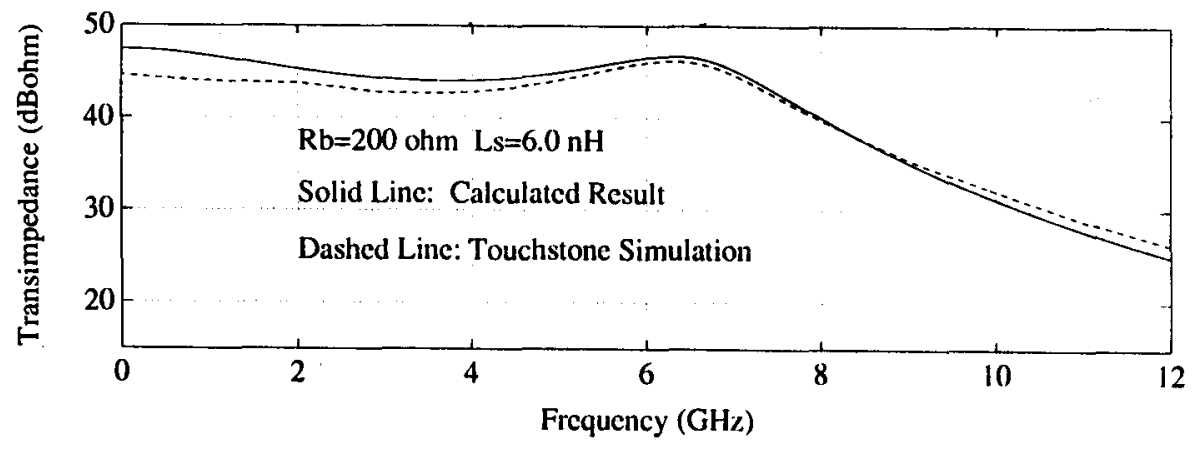

(a)

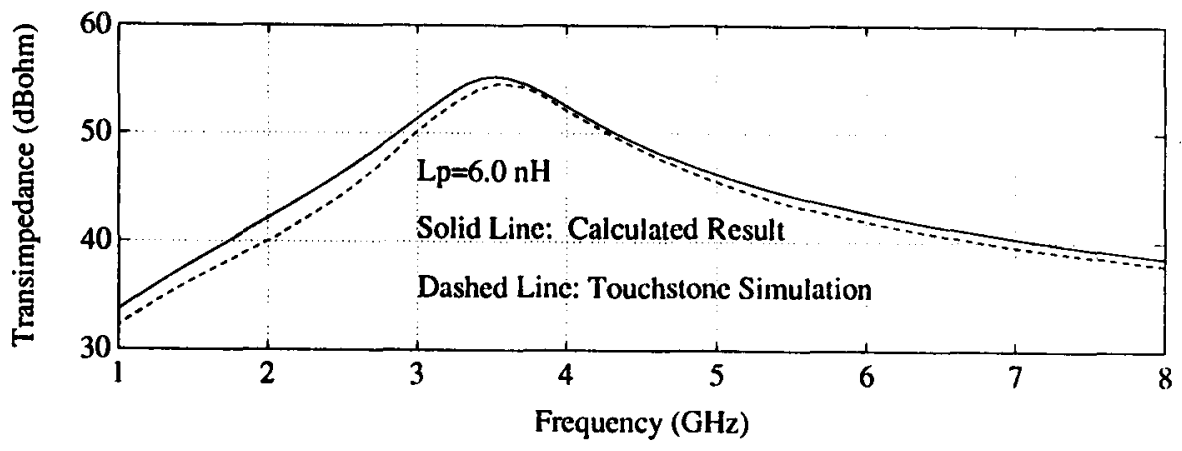

(b)

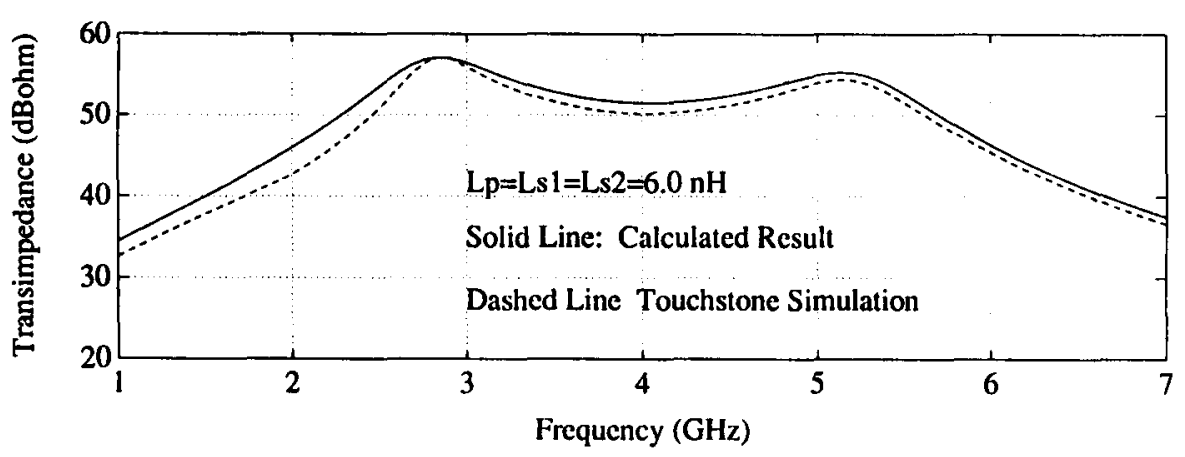

(c)

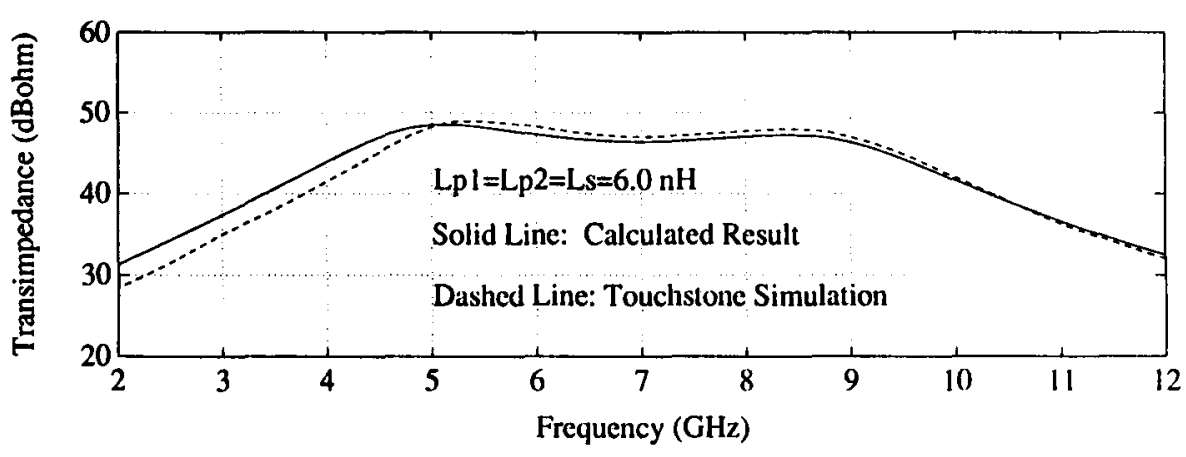

(d)

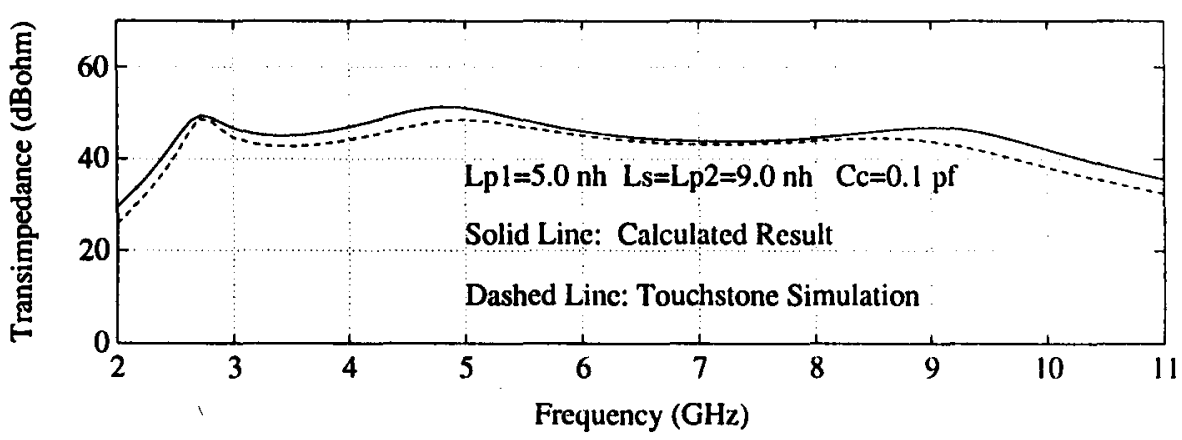

(e)

\section{Fig.3 Comparisons between the calculated and simulated transimpedances of the front ends with tuning network of (a) serially (b) parallel (c) Tequivalent transformer (d) $\pi$ equivalent transformer (e) 3rd order bandpass}




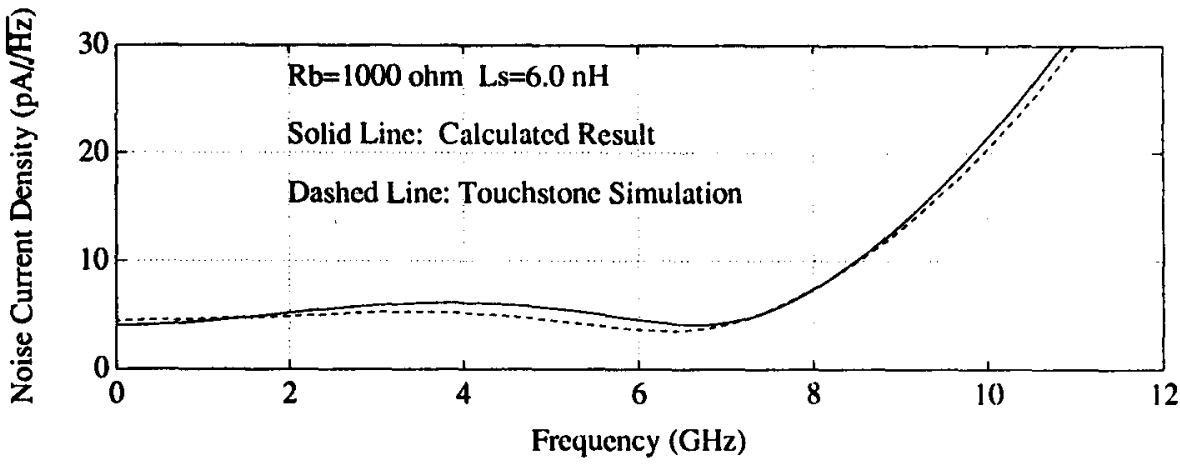

(a)

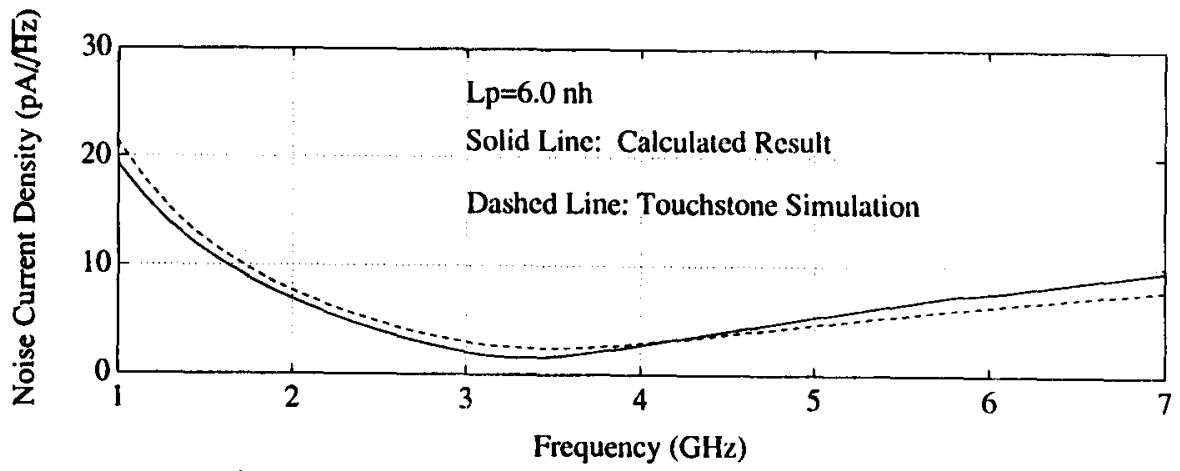

(b)
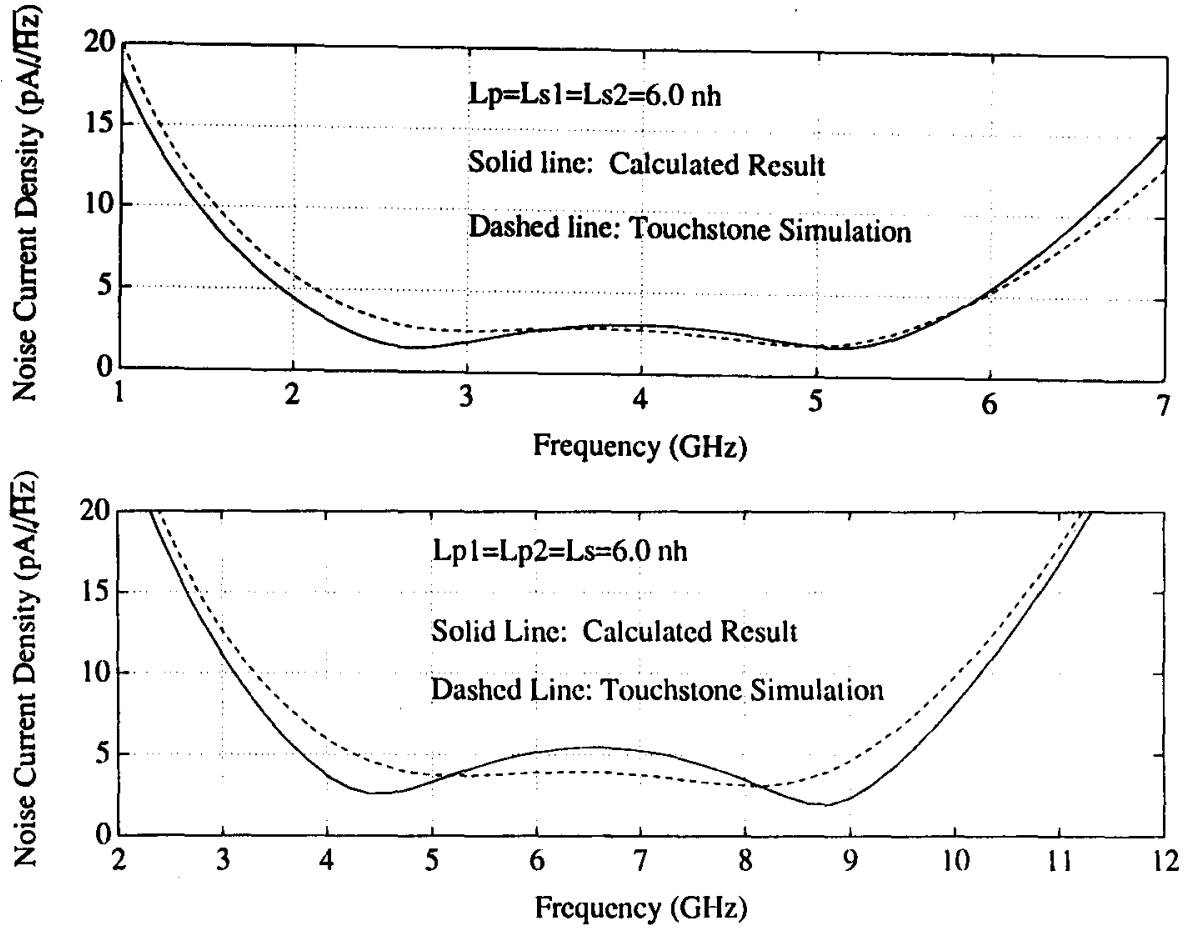

(d)

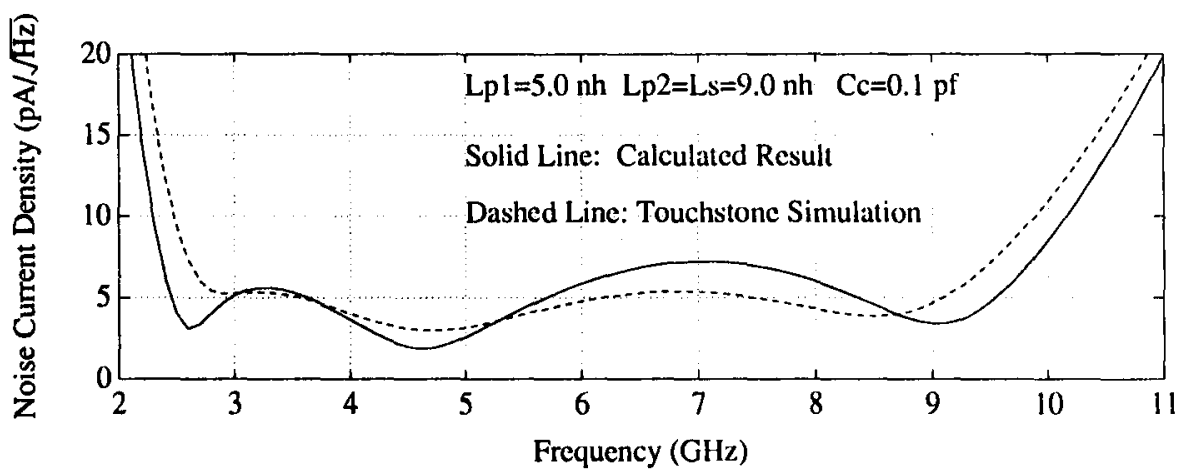

(e)

Fig.4 Comparisons between the calculated and simulated equivalent input noise current densities of the front ends with tuning network of (a) serially (b) parallel (c) T-equivalent transformer (d) $\pi$ equivalent transformer (e) 3rd order bandpass 